\title{
Electron collisions with molecules and molecular clusters ${ }^{\star}$
}

\author{
Jimena D. Gorfinkiel ${ }^{\mathrm{a}}$ \\ School of Physical Sciences, The Open University, Walton Hall, Milton Keynes MK7 6AA, UK
}

Received 31 October 2019 / Received in final form 10 January 2020

Published online 17 March 2020

(C) The Author(s) 2020. This article is published with open access at Springerlink.com

\begin{abstract}
State-of-the art computational studies of electron collisions with molecules and small molecular clusters are illustrated with results obtained from the application of the R-matrix method and the UKRMol/UKRMol+ suites. High-level calculations of electronic excitation cross sections and core-excited resonances, mainly of core-excited shape character, show excellent agreement with experiment for mid-size molecules like pyrimidine and thiophene. Simpler calculations are paving the way for an in-depth understanding of the effect of hydration on resonance formation: how the shift in resonance energy depends on the characteristics of the hydrogen bond and the resonance being studied. Finally, applications of the software to a little studied process, interatomic coulombic electron capture are also illustrated.
\end{abstract}

\section{Introduction}

The physics of electron scattering from molecules has been a focus of research in atomic and molecular physics for decades. These scattering processes possess both fundamental and applied interest [1]. Electron scattering experiments allow an insight into, for example, the electronic structure of molecules (e.g. [2]) as well as enabling the investigation of more fundamental phenomena like quantum coherence [3] and others [4]. From an applied perspective, the requirement to quantify and understand electron-molecule collisions stems from a number of fields and media [5,6]: from astrophysics to both natural and man-made plasmas (used, for example, in industry for microchip production) and processes induced by secondary electrons generated by ionizing radiation incident on biological (e.g. in humans subject to radiation-based medical treatment) and inorganic (e.g. detectors used in space missions [7]) matter.

The data requirements in particular have spurred the development of more sophisticated and accurate experimental and computational tools for the study of electron scattering from molecules. The advances in the computational investigation of these collisions have seen, over the last decade or so, the overhaul of many of the software tools employed, as well as the development of new ones. In particular, the interest in biological molecules, which are bigger and more electron rich, as targets has stimulated work to ensure the software is able to make use of current

* Contribution to the Topical Issue "Low-Energy Positron and Positronium Physics and Electron-Molecule Collisions and Swarms (POSMOL 2019)", edited by Michael Brunger, David Cassidy, Saša Dujko, Dragana Marić, Joan Marler, James Sullivan, Juraj Fedor.

a e-mail: J.Gorfinkiel@open.ac.uk computational capabilities; this has led, for example, to the parallelization of many programs (e.g. ePolyScat $[8,9]$, the Schwinger Multichannel with Pseudopotentials code [10] and the software suite used in this work) and the use of GPUs in some of them.

These developments have enabled scientist to study: (i) small targets (e.g. $\mathrm{H}_{2}$ [11]) with increasing level of detail, providing more accurate data than ever before for a number of scattering processes (vibrational and electronic excitation, elastic scattering, etc.); (ii) bigger targets than ever before (for example, radiosensitizers [12], biomass molecular fragments like lignin [13] and other biomolecules [14]) with a higher level of accuracy; (iii) the effect of the environment by means of the investigation of small molecular clusters. The latter strand of research emerges from the need to understand electron scattering processes beyond the gas phase, for example, in the cell (where low energy electrons are known to play a role in damage produced by ionizing radiation [15]). Clusters allow researchers to bridge the complexity gap between the gas and condense phases $[14,16]$ but are also interesting in themselves, for example in relation to atmospheric processes [17].

It is worth mentioning that the expertise and software used in electron scattering can also be applied, without the need of significant additional methodological or computational developments, to the study of other processes. One example is positrons collisions below the positronium formation threshold; another is interatomic coulombic electron capture (ICEC, see Sect. 3.3 for its definition), a process that takes place in atomic and molecular clusters. Finally, photoionization can be seen as half an electron scattering process: whereas in the latter there is both an incoming and outgoing unbound electron, in the case of photoionization, one needs to model only an outgoing unbound electron (after the deposition of energy by photons in the molecular system). This means that software 
to study electron scattering can be used for photoionization after a small number of additions. The software can not only be used to determine photoionization cross section and other observables, like the asymmetry parameter, but can generate data (transition dipole moments between a bound, normally the ground, state of the neutral molecule and the electronic continuum) that can be used [18,19] to study strong field processes (see, for example [20]).

In this paper, we describe some of the recent calculations performed using the R-matrix method and its software implementations for electron-molecule/cluster scattering (and positron scattering and photoionization). These examples describe: (i) state-of-the-art, highly accurate calculations of electronic excitation cross sections and core-excited resonances; (ii) simpler calculations aimed at understanding the effect of microhydration on shape resonance formation; (iii) an application to describe ICEC.

\section{The R-matrix method}

The R-matrix method and its application to electron scattering from molecules are well established, and a number of publications describe it in detail $[21,22]$. However, the computational implementation of the approach has changed significantly over the last 5 years with the development of the UKRmol+ suite [18]. Below, we provide a brief summary of the method and refer the reader to earlier publications for more details. We apply the approach within the fixed-nuclei approximation.

The R-matrix method is based on the division of space into inner and outer regions. The boundary between these regions is given by a sphere of radius a centred on the centre of mass of the system. In the inner region the scattering electron is indistinguishable from the target electrons and correlation and exchange effects play a crucial role whereas in the outer region they can be neglected. The radius $a$ must therefore be chosen such that the charge density of the electronic target states of interest is fully contained inside the R-matrix sphere, making the scattering electron distinguishable from the others when it is in the outer region.

In the inner region, a set of basis functions $\Psi_{k}$ determined by diagonalising the $\mathrm{N}+1$ non-relativistic hermitian Hamiltonian describing the system (hermiticity is ensured by addition of the Bloch operator [21]) is used to describe the system. Several levels of approximation are possible for $\Psi_{k}$ and these determine how they are constructed. In Static-Exchange/Static-Exchange plus Polarization (SE/SEP) calculations, only the ground electronic state of the molecule is considered and this is described at Hartree-Fock level. If electronic excitation is of interest and/or core-excited (see below) resonances are being investigated, the Close-Coupling approximation is used: in this case, the multiconfigurational wavefunctions of the ground and some excited states of the target go into building the $\Psi_{k}$ basis functions; these N-electron (target) wavefunction are usually, but not always, built using a Complete Active Space Self Consistent Field approach (CASSCF).

The basis functions $\Psi_{k}$ are used to construct the R-matrix at the boundary between regions. The outer region part of the problem is then solved by propagating this R-matrix [22] to an asymptotic distance, where the $\mathrm{K}$-matrix is determined by matching to known asymptotic expressions. The interaction potential between the scattering electron and the target is approximated in the outer region by a multipolar single-centre expansion that usually includes the dipolar and quadrupolar interactions.

Once the K-matrices are obtained, the cross sections can be calculated from the trivially determined T-matrices. In order to obtain resonance parameters (i.e. their energy and width) several approaches are possible; we use: (i) the diagonalization of the K-matrices to obtain the eigenphase sums that are then fitted to a Breit-Wigner profile; (ii) the calculation of the S-matrices and, from them, the timedelay matrices; fitting of the largest eigenvalues of the timedelay matrix (known as the time-delay) with a lorentzian function also provides the resonance parameters [23].

For the R-matrix calculations presented here we have mainly used the UKRmol+ suite, a re-engineered version of the UKRmol codes [24]. The use of the new suite was particularly necessary for the cluster studies, where, due to the size of the target, an $\mathrm{R}$-matrix radius $a=18 \mathrm{a}_{0}$ was required.

To calculate elastic and inelastic differential cross sections we used the program DCS [25].

\section{Results}

In this section we present some examples of state-of-theart and novel electron scattering calculations carried out using the R-matrix method.

\subsection{Resonances and cross sections for biomolecules}

Track structure modelling (of the effects of ionizing radiation in biological media) is used to assess how energy is deposited in the medium at the microscopic level. Cross section data for all processes induced by electron impact on molecules over a broad energy range are required for this purposes [26]. Data for elastic scattering are widely available (both in terms of integral and differential cross sections, although dipolar targets present some difficulties [27]) but electronically inelastic cross sections are harder to calculate and measure for low energy scattering [14].

Temporary anion states or resonances are crucial in low energy electron scattering: all scattering processes (elastic, vibrational and electronic excitation, neutral dissociation, dissociative recombination) can be enhanced by the presence of resonances. In addition, dissociative electron attachment (DEA) normally proceeds via resonance formation. Resonances linked to the electronic degrees of freedom are normally classified as shape (when they involve attachment to the molecule in the ground state) or coreexcited (when the electron transfers some of the energy to the molecule, exciting it electronically, as part of the attachment process); the core-excited resonances can, in turn, be classified as Feshbach (when they are energetically below the target electronic state identified as the parent state) or core-excited shape (when they are above). It is important to note that this classification is not "algorithmic": many 
molecules display the presence of resonances that are mixed in their character, for example, being partly shape and part core-excited. Resonances can also change their character as the geometry of the molecule changes [28].

In general, shape and core-excited shape resonances have shorter lifetimes (as they can decay to their parent states) and Feshbach resonances are narrower (i.e. have longer lifetimes). Again, this is just a general trend: narrow shape resonances are present in some molecules [28].

Resonances can be investigated experimentally for example, by measuring elastic or inelastic cross sections using electron transmission spectroscopy (ETS) or electron energy loss spectroscopy (EELS). Measuring anion yields due to DEA as a function of electron energy can also provide information about the resonances present in a molecular system. However, for larger molecules the resonance spectrum can be quite complex making it difficult to link specific calculated resonances to peaks in the anion production (unlike smaller molecules, where the assignation is usually more easily done). Velocity slice or map imaging experiments can provide additional information regarding the resonances that lead to DEA, particularly resonance symmetry, that facilitates comparison with theory [29]. Wider resonances are easier to detect if cross sections are being measured, so shape and core-excited shape resonances are more likely to be identified in this way.

From the computational point of view, shape resonances are the easiest to investigate as they only require describing the ground state of the molecule accurately. Conversely, describing core-excited resonance requires the (explicit or implicit) description of excited states of the target and careful modelling of electron correlation effects.

The R-matrix method and the UKRmol and UKRmol+ suites have been used to study core-excited resonances in a number of polyatomic molecules, from triatomics like water [30] to nucleobases like adenine and guanine [31]. However, experimental confirmation of the resonances identified in calculations has been scarce: ETS does not provide much information on core-excited resonances and EELS experiments for scattering energies below the ionization threshold are not abundant. Despite the fact that many DEA experiments have reported ion yields that are almost certainly linked to core-excited resonances, little is known about them.

Two examples of the predictive power of R-matrix calculations are given by the core-excited resonances in pyrimidine [32] and thiophene [33]. For these targets EEL spectra have confirmed, by measuring excitation functions and cross sections for electronic excitation to bands (for the former target) or specific states (for the latter), the presence of many of the resonances determined theoretically. Table 1 summarizes the resonances identified for pyrimidine both in calculations and measurements: we can see that the calculated results appear higher in energy and that differences between measured and calculated position increases with energy. This is a well known effect that is linked to an incomplete description of polarization effects in the R-matrix calculations [21,32].

Figure 1 shows the cross sections for excitation into the second $\left({ }^{3} \mathrm{~A}_{1}\right)$ excited state of thiophene for specific scattering angles (in other words, the excitation functions)
Table 1. Core-excited resonances of pyrimidine identified in R-matrix calculations and EEL spectra (see details in Regeta et al. [32]); note that the first resonance listed is actually of mixed shape and core-excited character. The widths of the calculated resonances are also provided.

\begin{tabular}{lccc}
\hline & \multicolumn{2}{c}{ Calculated } & EELS \\
\cline { 2 - 3 } Symmetry & $\mathrm{E}(\mathrm{eV})$ & $\Gamma(\mathrm{eV})$ & $\mathrm{E}(\mathrm{eV})$ \\
\hline${ }^{2} \mathrm{~B}_{1}$ & 4.78 & 0.38 & 4.35 \\
${ }^{2} \mathrm{~A}_{1}$ & 5.96 & 0.18 & - \\
${ }^{2} \mathrm{~A}_{1}$ & 6.15 & 0.18 & - \\
${ }^{2} \mathrm{~A}_{2}$ & 6.11 & 0.51 & 5.55 \\
${ }^{2} \mathrm{~B}_{1}$ & 6.37 & 0.58 & 5.55 \\
${ }^{2} \mathrm{~B}_{1}$ & 7.11 & 0.48 & 6.52 \\
${ }^{2} \mathrm{~A}_{2}$ & 7.33 & 0.43 & 6.52 \\
${ }^{2} \mathrm{~B}_{1}$ & 8.47 & 1.69 & 7.45 \\
${ }^{2} \mathrm{~B}_{1}$ & 12.3 & - & 10.3 \\
\hline
\end{tabular}

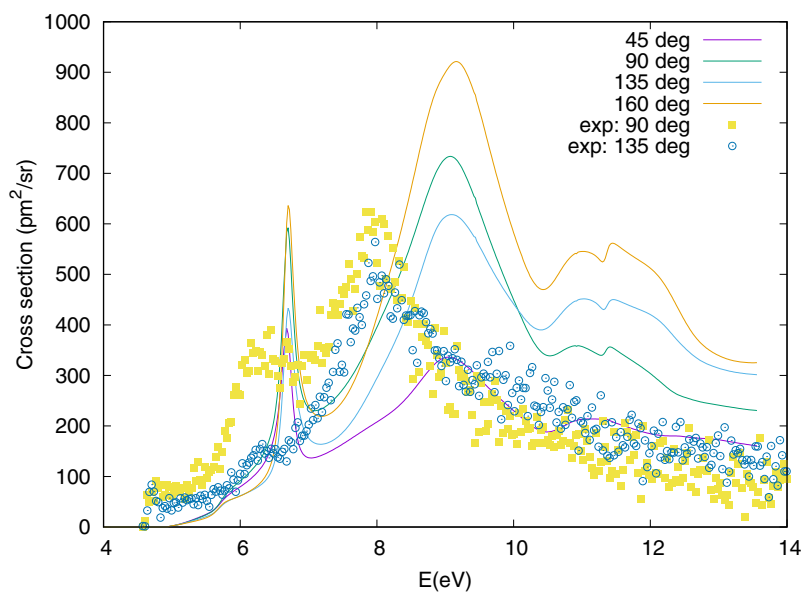

Fig. 1. Calculated (full lines) excitation functions for thiophene as a function of scattering energy (E) for the scattering angles indicated in the figure and the second excited state. The symbols correspond to EELS results for two of these angles. See [33] for details.

together with those measured for $90^{\circ}$ and $135^{\circ}$. Details of the calculation and experiment can be found elsewhere [33].

We can see that the size of the calculated cross section increases as the scattering angle goes from $45^{\circ}$ to $90^{\circ}$ but then decreases slightly for $135^{\circ}$ only to reach its maximum size for $160^{\circ}$. Two resonances are clearly visible in all cross sections: a narrow one at around $6.7 \mathrm{eV}$ and a wider one at around $9.2 \mathrm{eV}$. The variation with angle is smaller at lower energies (the size of the first resonant peak increases by around $50 \%$ between $45^{\circ}$ and $160^{\circ}$ ) and bigger at higher energies; the size of the second resonant peak increases by a factor of 3 between $45^{\circ}$ and $160^{\circ}$.

We also observe, for the two angles for which there is experimental data, that the size of both cross sections is almost identical, although the peaks are bigger in the calculated results. In addition, the energy dependence of the experimental results is well reproduced by the calculations: the two resonances discussed above are also visible in the experimental cross sections. The whole calculated 
cross sections look shifted to higher energies: this is due to the incomplete polarization description that leads to the shifting of the resonances, as described above.

\subsection{Effect of microhydration}

The study of small molecular clusters comprising one or several biomolecules and one or several water molecules is being pursued in order to bridge the gap in our understanding between gas phase and the processes that occur in the biological environment [14].

Using the R-matrix method and the UKRmol+ suite, extensive studies of pyridine- $\left(\mathrm{H}_{2} \mathrm{O}\right)_{n}$ and thymine- $\left(\mathrm{H}_{2} \mathrm{O}\right)_{n}$ with $n=1,2,3,5$ were performed $[34,35]$ at the SE level in order to understand the effect of microhydration on the two lowest $\pi^{*}$ shape resonances present in both ring molecules (see, for example, [36,37]). Earlier studies [38] using the Schwinger multichannel method (SMC) for the $\pi^{*}$ shape resonance of formic acid in clusters with 1 and 2 water molecules showed that: (i) the effect of water on the resonance position depended on whether $\mathrm{H}_{2} \mathrm{O}$ was the hydrogen donor/acceptor in the hydrogen bond, with the former leading to a lowering of the resonance energy and the latter to an increase; (ii) the effect was qualitatively additive.

Our aim was to determine whether these conclusions held for bigger molecules and whether the effect was quantitatively similar for different shape resonances of a given target. We interpreted our results by decomposing the of microhydration effect into indirect and direct effects. The indirect effect is due to the changes in the geometry of the hydrated molecule resulting from the formation of one or several hydrogen bonds. In general, these geometry changes are small, so this effect is usually smaller than the direct effect and can be quantified by calculating the resonance positions for the isolated molecule in two different geometries: the equilibrium one for the isolated molecule and the one the molecule has in the cluster equilibrium geometry (but without including the water molecules). Therefore, the calculated effect is dependent on the equilibrium geometry used in the calculations for the isolated molecule and the cluster.

The direct effect is due to the presence of the water molecules. This effect is quantified by performing calculations for the isolated molecule in the cluster geometry and for the cluster. The sum of both effects gives the resonance shift due to microhydration.

Table 2 shows the values of the shifts due to the indirect, direct and total effects for the pyridine- $\mathrm{H}_{2} \mathrm{O}$ cluster. In this cluster, the water molecule hydrogen bonds to the nitrogen atom in pyridine; it is therefore the hydrogen donor and we expect both resonances to be shifted to lower energies.

We can see that the indirect effect destabilizes both resonances (the shift is positive). This can be linked to the fact that many of the bond-lengths of pyridine are shortened in the cluster geometry [39]: the repulsive effects felt by the attached electron will be slightly stronger and this means the resonance energy will be slightly higher. The direct effect is stabilizing for both resonances and 3 to 4 times bigger than the indirect one.
Table 2. Energy shifts, in eV, for the first and second $\left(\pi^{*}\right)$ resonance in pyridine upon hydrogen bonding with a single water molecule calculated at SE level using the cc-pVDZ basis set (further details in [34]). The direct and indirect contributions and the total effect are listed. Negative values correspond to the resonance moving to lower energies in the cluster. See text for details.

\begin{tabular}{lccc}
\hline Resonance & Indirect & Direct & Total \\
\hline $1 \pi^{*}$ & 0.12 & -0.36 & -0.24 \\
$2 \pi^{*}$ & 0.08 & -0.31 & -0.23 \\
\hline
\end{tabular}

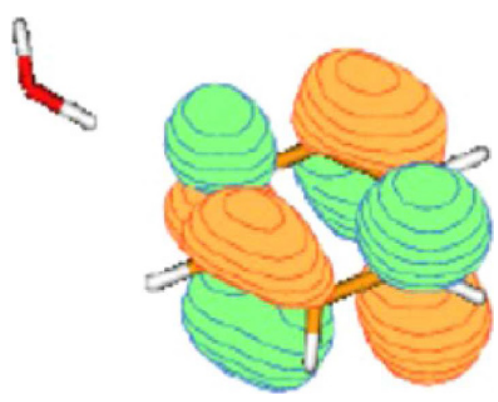

Fig. 2. Lowest energy unoccupied $\pi^{*}$ orbital in pyridine- $\mathrm{H}_{2} \mathrm{O}$. The orbital was determined with MOLPRO [40] in a SCF Hartree-Fock calculation using the cc-pVDZ basis set and a geometry optimized as described in [34].

One can see, looking at the orbitals occupied by the scattering electron in the two resonances being discussed (Figs. 2 and 3 respectively; the orbitals in the isolated molecule are practically identical) that the one involved in the first $\pi^{*}$ resonance describes an electronic density that is somewhat closer to the water molecule: one of the lobes of the orbital, centred on the nitrogen atom, points in its direction. This is consistent with the fact that the direct shift for this resonance is almost $20 \%$ bigger than for the second resonance; the orbital involved in the latter has no density on the nitrogen atom. The shape of the orbitals can also be linked to the relative size of the indirect shift: the $1 \pi^{*}$ orbital has density along two of the ring bonds, thus being more sensitive to changes in their length.

The shift due to the total effect is very similar for both resonances (although this is not always the case [39]) and leads to the stabilization of both of them, as expected given that $\mathrm{H}_{2} \mathrm{O}$ acts as the hydrogen donor. The shift for each resonance is also very similar to the energy change in the orbital involved in the resonance when going from the isolated molecule (in its equilibrium geometry) to the cluster.

We also confirmed the findings of Freitas et al., that there is a rough (qualitative) additivity of the effect as the number of water molecules in cluster increases [35]. In addition, we determined that there is a weak, but nonzero, dependence of the resonance shift on the binding site and that the stabilization/destabilization effect can be different for different resonances in the system.

Although both our [34] and the earlier SMC calculations performed both at SE and SEP level showed that similar conclusions were reached for both types of calculations, 


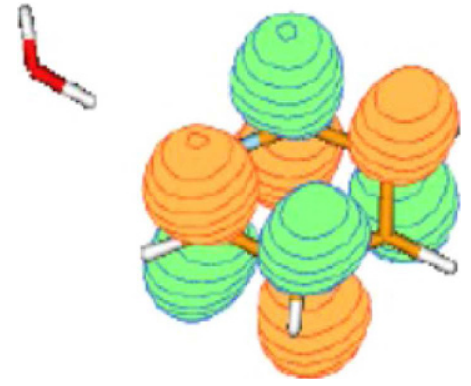

Fig. 3. Second lowest energy unoccupied $\pi^{*}$ orbital in pyridine$\mathrm{H}_{2} \mathrm{O}$. See caption of Figure 2 for details of the calculation.

inclusion of the polarization effects in a consistent way should provide a more accurate picture and improve our understanding of microhydration effects.

\subsection{Interatomic coulombic electron capture}

Interatomic coulombic electron capture is an electron induced process that has been predicted both theoretically $[41,42]$ and computationally [43] but not yet measured experimentally. In this process, an electron interacts with an heterogeneous (atomic or molecular) cluster in which one of the monomers is positively charged. The electron attaches to the cation, releasing some energy. This energy on its own is not sufficient for a different monomer (an atom or molecule with a higher ionization potential) in the cluster to ionise. However, if the sum of the kinetic energy of the electron and the attachment energy is larger than the ionization potential of the second monomer, ionization can take place. Taking as example a Neon cation immersed in a Helium cluster:

$$
\mathrm{Ne}^{+} @ \mathrm{He}_{n}+\mathrm{e} \rightarrow \mathrm{Ne} @ \mathrm{He}_{n}^{+}+\mathrm{e} .
$$

The energy of the ejected electron will be given by the difference between the ionization potential of the second monomer (He in this case) and the sum of the ionization energy of the first one $(\mathrm{Ne})$ and the initial kinetic energy of the electron. The process can therefore be seen as an inelastic scattering where the deposited energy causes the electron hole to "move" from one atom to another (although, more accurately, the process corresponds to attachment of one electron and emission of another). Experimentally, one could observe ICEC by measuring the "energy loss" of the free electron. Like microhydration, ICEC is a process in which the effect of the environment plays a role: however, whereas microhydration can enhance or quench [44] a process that occurs in the isolated monomer (e.g. DEA), in ICEC the environment is essential for it to take place.

The R-matrix method was used to determine the cross section for electron scattering from a $\mathrm{Ne}^{+} \mathrm{He}$ cluster. Close-coupling calculations based on a Hartree-Fock description of the ground state of $\mathrm{Ne}^{+} \mathrm{He}$ (in fact, three degenerate states corresponding to $\mathrm{Ne}^{+}\left(1 s^{2} 2 s^{2} 2 p^{5}\right)+$ $\left.\mathrm{He}\left(1 s^{2}\right)\right)$ and that of $\mathrm{NeHe}^{+}\left(\mathrm{Ne}^{+}\left(1 s^{2} 2 s^{2} 2 p^{6}\right)+\mathrm{He}\left(1 s^{1}\right)\right)$ were performed for a range of Ne-He distances $R$ between 3 and $10 \mathrm{a}_{0}$ (the equilibrium interatomic distance of $\mathrm{Ne}^{+} \mathrm{He}$ is around $\left.4 \mathrm{a}_{0}\right)$. The sum of the excitation cross sections between the degenerate states and the excited state give the ICEC cross section for $\mathrm{NeHe}^{+}$. However, experiments are more likely to involve bigger clusters and, for this reason, the ICEC cross section for $\mathrm{Ne}^{+} @ \mathrm{He}_{20}$ was calculated in the following way (see [43] for further details): vibrational wavefunctions for the cluster were determined from a variational quantum Monte Carlo calculation and 2000 geometries which sample the square of the wave function used in the computation of the ICEC cross section as the sum over all $\mathrm{NeHe}^{+}$pairs (i.e. with different $R$ values).

Even this simple calculation, where correlation effects are almost certainly underrepresented, yielded cross sections a few orders of magnitude higher than those for radiative recombination. Preliminary tests using more sophisticated wavefunctions for the states of the cluster indicate that the ICEC cross sections are likely to be even bigger. Calculated "energy loss" spectra for 5 and 10 eV scattering energy provide guidance for potential experiments.

\section{Conclusions}

Computational work on electron scattering from molecules and molecular clusters is providing both quantitative data and detailed insight into a number of collisional processes. For small molecular targets (few atoms and few electrons) results of a quality similar to that of atoms are being achieved. For medium-size targets, the full description of electronic correlation remains a challenge but quantitative agreement with experiment for the harder to determine excitation cross sections and core-excited resonances can be achieved.

Many challenges, nonetheless, still remain: modelling a number of processes of significant interest, like DEA and neutral dissociation (dissociative excitation) requires the inclusion of the nuclear degrees of freedom. Although progress has been made in this respect, polyatomic molecules remain mostly beyond current capabilities, unless simplifications that reduce the number of nuclear degrees of freedom are applicable.

Much remains to be understood of the effect of the environment on collisional processes. For example, more sophisticated calculations are needed to fully model the effect of microsolvation on resonance formation. In addition, effects like the transfer of kinetic energy into the vibrational modes of the cluster [45] need to be taken into account.

Finally, continued methodological and software developments will further improve our ability to describe and quantify electron scattering from molecules and clusters, contributing both to improving our understanding of fundamental molecular physics and to the description and modellization of other physical phenomena and electron interactions in condensed media.

I would like to acknowledge those colleagues that have contributed to the research presented here: Drs. Zdeněk Mašín, Agnieszka Sieradzka and Ms. Alexandra Loupas, Dr. Nicolas Sisourat and colleagues and Profs. Michael Allan and Gustavo Garcia and Dr. Khrystyna Regeta. 


\section{Author contribution statement}

Dr. J. D. Gorfinkiel wrote the manuscript based on research supervised by her and/or carried out in collaboration with researchers at various institutions.

Open Access This is an open access article distributed under the terms of the Creative Commons Attribution License (https://creativecommons.org/licenses/by/4.0/), which permits unrestricted use, distribution, and reproduction in any medium, provided the original work is properly cited.

\section{References}

1. S. Schippers, E. Sokell, F. Aumayr, H. Sadeghpour, K. Ueda, I. Bray, K. Bartschat, A. Murray, J. Tennyson, A. Dorn et al., J. Phys. B: At., Mol. Opt. Phys. 52, 171002 (2019)

2. M. Dubecký, R. Derian, L. Horváthová, M. Allan, I. Stich, Phys. Chem. Chem. Phys. 13, 20939 (2011)

3. E. Krishnakumar, V.S. Prabhudesai, N.J. Mason, Nat. Phys. 14, 149 (2018)

4. F.H. Ómarsson, E. Szymanska, N.J. Mason, E. Krishnakumar, O. Ingólfsson, Phys. Rev. Lett. 111, 063201 (2013)

5. K. Bartschat, J. Phys. B: At., Mol. Opt. Phys. 51, 132001 (2018)

6. O. Ingólfsson, Low-Energy Electrons (Jenny Stanford Publishing, New York, 2019)

7. Y. Liu, T. Zhu, J. Yao, X. Ouyang, Sensors 19, 1767 (2019)

8. F.A. Gianturco, R.R. Lucchese, N. Sanna, J. Chem. Phys. 100, 6464 (1994)

9. A.P.P. Natalense, R.R. Lucchese, J. Chem. Phys. 111, $5344(1999)$

10. R.F. da Costa, M.T.d.N. Varella, M.H.F. Bettega, M.A.P. Lima, Eur. Phys. J. D 69, 159 (2015)

11. M. Zawadzki, R. Wright, G. Dolmat, M.F. Martin, L. Hargreaves, D.V. Fursa, M.C. Zammit, L.H. Scarlett, J.K. Tapley, J.S. Savage et al., Phys. Rev. A 97, 050702 (2018)

12. L.M. Cornetta, F. Kossoski, M.T.d.N. Varella, J. Chem. Phys. 147, 214310 (2017)

13. M.A. Ridenti, J.A. Filho, M.J. Brunger, R.F. da Costa, M.T.d.N. Varella, M.H. Bettega, M.A. Lima, Eur. Phys. J. D 70, 161 (2016)

14. J.D. Gorfinkiel, S. Ptasińska, J. Phys. B: At., Mol. Opt. Phys. 50, 182001 (2017)

15. B. Boudaïffa, P. Cloutier, D. Hunting, M.A. Huels, L. Sanche, Science 287, 1658 (2000)

16. J.D. Gorfinkiel, S. Caprasecca, in Radiation Damage in Biomolecular Systems, edited by G. García Gomez-Tejedor, M.C. Fuss (Springer Netherlands, 2012), pp. 127-142

17. J. Lengyel, J. Fedor, M. Fárník, Phys. Chem. Chem. Phys. 21, 8691 (2019)

18. Z. Mašín, J. Benda, J.D. Gorfinkiel, A.G. Harvey, J. Tennyson, Comput. Phys. Commun. 249, 107092 (2020)

19. A.C. Brown, G.S. Armstrong, J. Benda, D.D. Clarke, J. Wragg, K.R. Hamilton, Z. Mašín, J.D. Gorfinkiel, H.W. van der Hart, Comput. Phys. Commun., https://doi. org/10.1016/j.cpc.2019.107062

20. D.R. Austin, F. McGrath, L. Miseikis, D. Wood, P. Hawkins, A.S. Johnson, M. Vacher, Z. Mašín, A. Harvey, M. Ivanov et al., Faraday Discuss. 194, 349 (2016)

21. J. Tennyson, Phys. Rep. 491, 29 (2010)

22. P.G. Burke, R-Matrix Theory of Atomic Collisions: Application to Atomic, Molecular and Optical Processes (Springer, 2011)

23. F.T. Smith, Phys. Rev. 118, 349 (1960)

24. J.M. Carr, P.G. Galiatsatos, J.D. Gorfinkiel, A.G. Harvey, M.A. Lysaght, D. Madden, Z. Mašín, M. Plummer, J. Tennyson, H.N. Varambhia, Eur. Phys. J. D 66, 58 (2012)

25. Z. Mašín, DCS:a Program to Generate Orientationaveraged DCS for Electronically Elastic and Inelastic Collisions (2018), https://gitlab.com/Masin/DCS

26. A. Muñoz, M.C. Fuss, M.A. Cortés-Giraldo, S. Incerti, V. Ivanchenko, A. Ivanchenko, J.M. Quesada, F. Salvat, C. Champion, G.G. Gómez-Tejedor, in Radiation Damage in Biomolecular Systems, edited by G. García Gómez-Tejedor, M.C. Fuss (Springer, Netherlands, Dordrecht, 2012), pp. 203-225

27. A.I. Lozano, A. Loupas, F. Blanco, J.D. Gorfinkiel, G. Garcia, J. Chem. Phys. 149, 134303 (2018)

28. A. Loupas, J.D. Gorfinkiel, Phys. Chem. Chem. Phys. 19, $18252(2017)$

29. I.I. Fabrikant, S. Eden, N.J. Mason, J. Fedor, in Advances in Atomic, Molecular, and Optical Physics, edited by C.C.L. Ennio Arimondo, S.F. Yelin (Academic Press, 2017), Vol. 66, pp. 545-657

30. J.D. Gorfinkiel, L.A. Morgan, J. Tennyson, J. Phys. B: At., Mol. Opt. Phys. 35, 543 (2002)

31. A. Dora, L. Bryjko, T. van Mourik, J. Tennyson, J. Chem. Phys. 136, 024324 (2012)

32. K. Regeta, M. Allan, Z. Mašín, J.D. Gorfinkiel, J. Chem. Phys. 144, 024302 (2016)

33. A. Loupas, K. Regeta, M. Allan, J.D. Gorfinkiel, J. Phys. Chem. A 122, 1146 (2018)

34. A. Sieradzka, J.D. Gorfinkiel, J. Chem. Phys. 147, 034302 (2017)

35. A. Sieradzka, J.D. Gorfinkiel, J. Chem. Phys. 147, 034303 (2017)

36. A. Dora, L. Bryjko, T. van Mourik, J. Tennyson, J. Phys. B: At., Mol. Opt. Phys. 45, 175203 (2012)

37. A. Sieradzka, F. Blanco, M.C. Fuss, Z. Mašín, J.D. Gorfinkiel, G. García, J. Phys. Chem. A 118, 6657 (2014)

38. T.C. Freitas, K. Coutinho, M. Varella, M.A.P. Lima, S. Canuto, J. Chem. Phys. 138, 174307 (2013)

39. A. Sieradzka, Ph.D. thesis, The Open University, 2017

40. H.J. Werner, P.J. Knowles, R. Lindh, F.R. Manby, M. Schütz et al., MOLPRO, version 2009.1, a package of ab initio programs (2009)

41. K. Gokhberg, L.S. Cederbaum, J. Phys. B: At., Mol. Opt. Phys. 42, 231001 (2009)

42. K. Gokhberg, L.S. Cederbaum, Phys. Rev. A 82, 052707 (2010)

43. N. Sisourat, T. Miteva, J.D. Gorfinkiel, K. Gokhberg, L.S. Cederbaum, Phys. Rev. A 98, 020701 (2018)

44. J. Kočišek, A. Pysanenko, M. Fárník, J. Fedor, J. Phys. Chem. Lett. 7, 3401 (2016)

45. M. McAllister, N. Kazemigazestane, L. Henry, B. Gu, I. Fabrikant, G. Tribello, J. Kohanoff, J. Phys. Chem. B 123, 1537 (2019) 